\title{
Die Couch im Isolationszimmer - Psychoanalytisches Denken und psychiatrische Institutionen ${ }^{1}$
}

\author{
Michael Niebler und Simon Mettler (Zürich)
}

Zusammenfassung: Der Artikel befasst sich mit der Frage, in welchem Verhältnis psychoanalytisches Denken und psychiatrische Kliniken heute stehen. Unter Rückgriff auf einen Maschinenbegriff, der mehr meint als konkrete technische Objekte, wird die Klinik zunächst als Einrichtung beschrieben, die der Symbolisierung von Unbewusstem dient. Diese abstrakten Maschinen sind gekennzeichnet durch immer schon vorhergehende Verbindungen gerade von offensichtlich Unverbundenem. Massgeblich für das Funktionieren von Klinik als Symbolisierungsmaschine ist das Aufgreifen und Auswerten von Irritationen, die im Kontrast zu einem geregelten Ablauf stehen und erst vor dessen Hintergrund gesehen werden können. Demgegenüber steht die Tendenz, Kliniken möglichst effizient und reibungslos zu betreiben, was sie aber ihren tatsächlichen Wert einbüssen lässt. Die Klinikmaschine ist aber auch wichtige Ordnungsmacht und markiert die Grenzen des Therapeutischen. In dieser Funktion wird therapeutisches Arbeiten ausserhalb der Klinik erst ermöglicht. Psychiatrische Kliniken sind für Therapeuten immer Ort und Nicht-Ort zugleich, zu denen man immer im Bezug steht. Wird man sich dieser maschinischen Verbindung zur Klinik bewusst, entsteht die Möglichkeit, diese reflexiv zu nutzen. Deshalb plädieren die Autoren für eine stärkere Verknüpfung praktizierender Psychoanalytiker mit psychiatrischen Institutionen, um deren Funktionieren anhand von Irritationen zu gewährleisten.

Schlüsselwörter: Klinik, Wunschmaschine, Zwangsbehandlung 
All wüssed all wahre Gründ

S'chunnt scho guet

und grad drum lupfts dir jetzt dänn dä Huet

Was choschtet än Fründ

Ich han zwei Bier und zwei Ohre

Ich glaub es isch no nüt verlore.

(Mettiwetti, 2013)

Zurückgelehnt Zuhören, als würde man gerade entspannt ein Glas Bier geniessen: Das ist die rechte psychoanalytische Haltung. Zumindest wird dies von Fritz Morgenthaler so kolportiert (Sylvia von Arx, mündliche Mitteilung). Die Autoren dieses Artikels üben sich gerne in dieser Haltung, wenn sie gemeinsam über ihre Lebenswelt nachdenken und sich mit einem Bier in der Hand dabei zuhören. Für beide, Simon Mettler als Leiter mehrerer psychiatrischer Akutstationen und Michael Niebler als (Notfall-)Psychiater in eigener Praxis, ist die psychiatrische Klinik ein wichtiger Ort - ein Bezugspunkt, über den im Folgenden nachgedacht werden soll. Die Darstellungsform ist entgegen der Gepflogenheiten eines Fachartikels angelegt wie ein Dialog. Das ist zum einen eine Würdigung der oben beschriebenen Haltung und zum anderen Ausdruck des Inhalts. Beide Autoren machten die Erfahrung, als Psychoanalytiker in der heutigen Kliniklandschaft teilweise wie Exoten behandelt zu werden, sodass sie sich fragten, in welchem Verhältnis ihre geliebte Psychoanalyse zu den wichtigen psychiatrischen Institutionen steht. Der Versuch, diese Frage aufzufächern und zu konkretisieren brachte das Konzept der Klinik als Symbolisierungsmaschine hervor (Niebler, 2015, S. 12 ff.). Darunter ist eine bestimmte Form von Maschine (im Folgenden abstrakte Maschine genannt) zu verstehen, die die Möglichkeit hat Unbewusstes zu symbolisieren, und zwar durch die Verbundenheit und das Zusammenwirken der Menschen im Einflussbereich einer psychiatrischen Klinik. Diese abstrakten Maschinen sind in Abgrenzung von konkreten Maschinen besser zu verstehen, wobei letztere wie technische Objekte funktionieren. Während eine konkrete Maschine immer demselben programmierten Ablauf folgt (z. B. auf Schalter an folgt Licht geht an, auf Schalter aus folgt Licht geht aus), stellt eine abstrakte Maschine Verbindungen gerade zwischen Unverbundenem her, in diesem Fall zwischen vorher un-verstehbarem Unbewussten und Symbolen. Neben diesem Konzept tat sich aber auch zwischen «Kliniker» und «Zuweiser» ein Spannungsfeld auf, das wie ein Ping-Pong-Spiel zwischen den vermeintlich so unterschiedlichen Positionen hin- und hergeht. Den Einstieg in dieses Spiel, das 
auch ein Beziehungsgeflecht symbolisiert, bildet die Sorge des Niedergelassenen um einen Patienten.

Michael Niebler: Ich stecke in einem Dilemma, was die Klinik betrifft. Ich habe einen jungen Mann gegen seinen Willen eingewiesen und es treibt mich um, ob ich ihm damit etwas Gutes getan habe. Ich hatte Dienst und wurde von den Betreuern einer Wohngemeinschaft für psychisch kranke Menschen gerufen. Der junge Mann wohnte noch nicht lange dort. Er war vor kurzem, nachdem er sich ins Ausland abgesetzt hatte, zurück in die Schweiz gebracht worden. Dass er offenbar psychotisch war, entnahm ich aber vor allem den Schilderungen der Betreuerin. Er selbst sagte mir nur einzelne Worte, die aber zu dem passten, was ich gehört hatte: Die Polizei des Landes, in dem er war, werde kommen und ihn holen. Mir wurde gesagt, er habe am Vorabend randaliert und die Mitbewohner seien aus Angst weggegangen. Ich ging in Begleitung einer Betreuerin und zweier Polizisten in die Wohnung. Im Nachhinein muss ich gestehen, dass das Urteil einer Zwangseinweisung schon vorher gefällt war. Ich hatte gehört, die Klinik würde ihn ohnehin schon erwarten, da die behandelnde Psychiaterin in der zuständigen Institution arbeite und einen Klinikeintritt vorbereitet hatte. Ich hatte Angst, und ein offenes Gespräch kam nicht zustande. Auch wenn ich wohl formal das Richtige getan habe, also mich juristisch nicht angreifbar wähne, so frage ich mich doch, ob ich für den jungen Mann etwas Gutes getan habe. Ich fühle mich ein Stück weit, als hätte ich einfach eine «ordnungspolizeiliche Funktion» (Gaebel \& Zielasek, 2011, S. 81) für die Klinikmaschine erfüllt.

Simon Mettler: Ein gar nicht so untypischer Fall, wie mir scheint. Schon bei den Stichworten Psychose, junger Mann, Auslandaufenthalt und Fürsorgerische Unterbringung sind mir spontan sicher zwei Patienten in den Sinn gekommen. Würde ich noch etwas länger überlegen, kämen wohl noch einige dazu. Das ist nicht verwunderlich, insofern Deine Idee von der Klinikmaschine ja am ehesten eine Idee im Sinne Walter Benjamins darstellt, d.h. die Beschreibung einer Konstellation, die durch ihre Extreme bestimmt ist (2011, S. 771). Entsprechend erinnert man sich an einige besonders beeindruckende weil extreme Fälle, durch die man in der Lage ist ein Grundprinzip zu begreifen. Vermutlich geht's Dir nicht anders und doch ist dieser vermeintlich klare Fall alles andere als gerade das, weil Dich etwas daran irritiert. Ein Dilemma der Art wie man «das Richtige» (Rechte) und/oder «das Gute» tun kann und wie sich das eine zum anderen verhält. Nun könnte ich Dich mit dem Verweis auf vergleichbare Fälle mit guten Verläufen beschwichtigen. Dir mit Hilfe einer Verallgemeinerung einen Ausweg aus dem Dilemma weisen. Eine Objektivierung mit Heilsversprechung, wenn Du bereit 
wärst, den Preis in Form der Aufgabe oder Verdrängung Deiner Subjektivität zu zahlen. Im psychoanalytischen Sinne erkenntnisreicher und allenfalls auch lustvoller ist jedoch gerade die Bereitschaft, sich von dem, was ist, irritieren zu lassen (Kirchhof, 2015, S. 20). Es kommt mir vor, als stünde in Deiner Schilderung «das Richtige» für «das Objektive» und «das Gute» für «das Subjektive» und ich befürchte, dass die Dinge so einfach nicht liegen. Hier möchte ich versuchen etwas zu klären, auf die Gefahr hin, die Irritation zu vergrössern. Die Fürsorgerische Unterbringung (FU) ist im Zivilgesetzbuch im Artikel 426 geregelt (vgl. ZGB2019). Darin heisst es, dass eine Person, die an einer psychischen Störung leidet, in einer geeigneten Einrichtung untergebracht werden darf. Als notwendige Bedingung wird festgehalten, dass die nötige Behandlung oder Betreuung nicht anders erfolgen kann. In den Bestimmungen des Kantons Zürichs ist zudem festgehalten, dass dies als "ultima ratio» nur zulässig ist, wenn keine weniger einschneidende Massnahme der betroffenen Person genügenden Schutz bietet und nur, wenn eine geeignete Einrichtung zur Verfügung steht (Kantonsärztlicher Dienst, 2012). Rechtlich hast Du bis hierhin nichts zu befürchten und doch wird klar, dass hier kein reiner Automatismus beschrieben wird, schliesslich ist es eine Massnahme, die getätigt werden «darf» und nicht «muss». Hier eröffnet sich ein nicht unerheblicher Spielraum. Auch wenn die viel genannte Selbst- oder Fremdgefährdung im Gesetzestext nicht auftaucht, so ist laut ZGB die Belastung und der Schutz von Dritten immerhin «zu berücksichtigen», was Dich juristisch absichert. Die beruhigende juristische Unangreifbarkeit scheint Dich insofern zu beunruhigen, als dass Du das Gefühl hast, nicht wirklich eine Entscheidung getroffen zu haben, sondern vor vollendete Tatsachen gestellt worden zu sein. Interessant, dass, sich auf die Institution (in diesem Fall den Rechtsstaat) zu berufen, Dich in diesem System absichert, Dir Macht verleiht, Dich in Deinen Entscheidungen einschränkt und Dir gleichzeitig einen Handlungsspielraum eröffnet. Auf diese Weise kannst Du im System als Subjekt (auch in der Klinikmaschine) existieren, aber natürlich nur, wenn die Entscheidungen, wie in Deinem Beispiel, nicht schon im Voraus getroffen worden wären. Das stellt Dich vor eine Schwierigkeit, welche Deinem ausgeführten Dilemma vorangeht. Wenn Du den Entscheid zur Fürsorgerischen Unterbringung nicht getroffen hättest, wäre mit grosser Wahrscheinlichkeit ein anderer Arzt angefragt worden, diesen Nichtentscheid zu fällen. Nun hast Du Dich dafür entschieden die FU auszustellen, hattest aber keine Wahl. Ich frage mich, was das mit unserem Patienten zu tun hat. Vielleicht, dass es ihm ganz ähnlich ergeht wie Dir. Immerhin beruft auch er sich in seinem Wahn auf eine höhere Instanz (die Polizei), welche ihn in seinem System insofern absichert, als 
er auf diese Weise existiert. Rom beschreibt die Verfolgung mit Bezug auf Mentzos (2004) als «Existenzgarantie des Schizophrenen» (Rom, 2007, S. 67). Es gilt die Verfolgung zu bewahren. Sie ist trotz oder eben gerade wegen der Bedrohung mit Vernichtungsangst seine Existenzgarantie (ebd.). Er hat also Macht, insofern als sich die Mitbewohner und Du vor ihm fürchten und ist gleichzeitig in seinen Entscheidungen eingeschränkt. Er ist auf der Flucht und lässt sich zwar vorerst nicht einmal durch Landesgrenzen stoppen, wird am Ende aber gegen seinen Willen in die Klinik eingewiesen. Psychose ist also möglicherweise auch die Frage, ob Du darüber sprechen kannst. Du und Dein Patient seid also zum besagten Zeitpunkt in einer ähnlichen Situation, getrennt durch die Sprachlosigkeit. Allerdings befindest Du Dich beinahe zufälligerweise auf der Seite mit mehr Realitätsbezug, was dazu führt, dass Deine Handlungen mehr Einfluss auf ihn haben als umgekehrt. Die Frage sei erlaubt, ob gerade dem Umstand der Zufälligkeit Dein Unbehagen geschuldet sein könnte. Du zweifelst; hast Du ihn nun gerettet oder vernichtet? Jetzt kannst Du über das Vorgefallene berichten. Ist das gegebenenfalls der nicht unwesentliche, um nicht zu sagen der entscheidende Unterschied zwischen Dir und Deinem Patienten?

The man in me will hide sometimes, to keep from being seen. But that's just because he doesn't wanna turn into some machine. (Bob Dylan, 1970)

MN: Bei mir regt sich zunächst Widerstand: Es soll Zufall sein, wer psychotischer und weniger psychotisch durch die Welt geht? sagt der «Halbgott in Weiss» in mir, aber so ich diese «Identifikation mit der Rolle» (Parin \& Parin-Matthèy, 1978, S. 415) hinter mir lassen und zum Kontakt des Patienten mit mir zurückkehren kann, muss ich Dir Recht geben. In der Situation mit der Polizei wortwörtlich an der Türe war es wohl unmöglich zu sprechen. Insofern war es auch gut, auf die Klinik zurückgreifen zu können, die Sprechen wieder ermöglichen kann, was ich als eine Eigenschaft der Klinikmaschine beschreiben möchte. Es ist naheliegend, eine moderne psychiatrische Klinik als Maschine zu beschreiben. Umso mehr, als dort mittlerweile die Behandlungen mit Ablaufdiagrammen standardisiert werden (Niebler, 2015, S. 3) . In diesen wird festgelegt, wer wann involviert wird, wer was zu tun hat und was das Ergebnis sein muss. Diese Abläufe werden graphisch wie das Schaltdiagramm einer Maschine dargestellt. Kliniken können also wie technische Objekte funktionieren mit festgelegten immer gleichen Abläufen. Das führt jedoch nicht weiter, wenn man die grösseren Zusammenhänge betrachtet, 
in denen psychiatrische Kliniken stehen. Für Patienten und deren Angehörige ist es ein Ort der Hoffnung, der Genesung, aber auch von Einschränkung und im Extremfall des Zwangs. Für Psychotherapeuten und andere Berufe dieses Bereichs kann es Arbeitsplatz, Weiterbildungsstelle, aber auch ein zweifelhafter Ort sein. Denn ist nicht zu befürchten, dass die Prozesse und Vorschriften einer gut geölten Klinikmaschine zum Selbstzweck geraten und die Begegnung mit den Menschen darunter verschwindet? Die Kliniken erfüllen Aufträge von Versicherungen, Sozialinstitutionen und Behörden, geniessen sogar hoheitliche Rechte. Zusammenfassend ist die Klinik ein auf mehreren Ebenen kompliziert vernetzter Komplex, hin zu Menschen, aber auch zu Institutionen. Zunächst zurück zu den konkreten Maschinen, die einen Aspekt beschreiben, wie Kliniken aus Sicht eines betriebswirtschaftlich denkenden Managers geführt werden. Dort steht die Funktion im Vordergrund. Eine Maschine wird auf einen Zweck hin konstruiert, der als erfüllt oder nicht-erfüllt gemessen werden kann. Es würde also darum gehen, einen Patienten an die Klinik anzukoppeln und gesünder abzukoppeln, nachdem er durch einen geplanten Ablauf, genannt Therapieprogramm hindurchbewegt wurde. Dabei sehe ich aber eine grosse Gefahr der Funktionalisierung der Menschen in der Klinikmaschine, egal auf welcher Seite, ob es nun den jungen Mann mit der Psychose betrifft oder Dich als Kliniker. Zudem geht das grosse Potential verloren, das in der Klinik als abstrakte Maschine steckt. Mit dieser greife ich auf einen Begriff zurück, der u.a. von Gilles Deleuze und Félix Guattari im AntiÖdipus als Wunschmaschinen wiederbelebt (2014, S 503 ff.) und von Gerald Raunig auf soziale Bewegungen bezogen wurde (2008, S. 27 ff). Ursprünglich war mit (altgriechisch) mechané Mittel, List oder Vorrichtung gemeint, unabhängig davon, ob es materiell oder immateriell war (Raunig, 2008, S. 32). Es war also sowohl das trojanische Pferd als Holzkonstruktion als auch die damit verbundene List gemeint. Ein weiteres Beispiel wäre der im Theater vorkommende deus ex machina: Wenn sich die Helden des Dramas in eine unlösbare Situation gebracht haben, erscheint ein Gott, dargestellt von einem Schauspieler, der durch eine Seilkonstruktion auf die Bühne herabgelassen wird. Deus ex machina meint sowohl die Seil-Vorrichtung als auch die erzählerische Wendung. Entsprechend wird nicht unterschieden zwischen dem Menschen, der einen Zweck erreichen will, und dem dafür verwendeten (funktionalisierten) Werkzeug. Abstrakte Maschine meint vielmehr eine schon immer vorausgehende Verbindung, ein Strömen zwischen Konnexionen, uneingeschränkt von festen Symbolisierungsregelungen. Das «Maschinenphylum» (Deleuze \& Guattari, 2014, S. 503) ist ein Stamm, der stets schon vorhanden, stetig verbindet, was getrennt erscheint. Ein Band gerade zwischen dem, was unverbun- 
den scheint. Und gerade hier liegt das Potential der Klinik: Sie kann genutzt werden, um Unverbundenes in Verbindung zu bringen: Symptome mit Sprache, unbedachtes Agieren mit Begriffen, Gedanken mit Emotionen. Die Klinik ist ihrem Wesen nach eine Symbolisierungsmaschine, da sie dazu geschaffen wurde Symptome unter Subtraktion des Individuellen in ein System einzuordnen (Foucault, 2011, S. 24). Man könnte also von einer Sonderform einer abstrakten Maschine sprechen, die durch ihre Funktion eine bestimmte Ausrichtung erfährt. Nun gibt es aber eine Art dieses Klinikbetriebs, die eine offen forschende Ausrichtung ins Gegenteil verkehrt. Nicht mehr ein Interesse für das Symptom des Einzelnen und dessen mögliche Implikationen und Ursachen stehen im Vordergrund, sondern die Einordnung in ein starres System von schon Bekanntem. Mitarbeiter und Patienten werden einem Diktat unterworfen. Damit meine ich z. B. die gängige Praxis, aufgrund einer Diagnose eine leitliniengestützte Therapie zu initiieren. Eine Momentaufnahme aus dem Leben eines Menschen wird in eine Diagnose übertragen, aus der dann gemäss einer Regel die mutmasslich wirksame Therapie abgeleitet wird. Der lebensgeschichtliche Kontext, in dem das Symptom steht, muss dabei ausgeblendet werden. Entsprechend ist man nicht mehr frei darin, sich dafür zu interessieren, was damals und heute passiert(e) und wie sich das im aktuellen Beziehungsnetz niederschlägt. In der Klinik werden dabei Patienten und Mitarbeiter mittels des gleichen Mechanismus gezwungen, auf das Wahrnehmen des Individuellen zugunsten eines vorgegebenen Wissens $\mathrm{zu}$ verzichten. Und diese Funktionalisierung der Menschen besorgt mich. Gleichzeitig sind Regeln und Ordnungssysteme nötig, um die Existenz einer Institution zu garantieren. Und die psychiatrische Klinik hat eben auch das Potential, als Symbolisierungsmaschine zu funktionieren und damit ansonsten Unverständliches zugänglich zu machen. Es besteht also die Gefahr eines dialektischen Umschlags: Die Ordnungssysteme, die eine Klinik und damit eine Symbolisierungsmaschine erst möglich machen, können zum Selbstzweck geraten und dabei die weitere Fähigkeit zur Symbolisierung verunmöglichen.

Pure Vernunft darf niemals siegen

Wir brauchen dringend neue Lügen

Die uns den Schatz des Wahnsinns zeigen

Und sich danach vor uns verbeugen

Und die zu Königen uns krönen

Nur um uns heimlich zu verhöhnen. (Lowtzow, 2008) 
SM: Als «Kliniker» angesprochen werde ich versuchen, Dich bezüglich Deiner Ängste zu beruhigen, zweifle aber, ob es mir gelingen wird. Wenn ich Dich richtig verstehe, zweifelst Du daran, dass es der Klinik als Institution gelingt, als abstrakte Maschine konzipiert, die von Dir gewünschte Symbolisierungsleistung zu erbringen. Du befürchtest eine Behandlung nach Schema. Ein psychopathologischer Befund wird erhoben, auf dessen Basis wird eine Diagnose gestellt und diese leitliniengemäss mit einem oder mehreren Medikamenten behandelt. Die Symptomreduktion gibt Aufschluss über den Erfolg der Behandlung und dieser Vorgang wird schliesslich in einem knappen Austrittsbericht festgehalten. Die Krankheit wird erfasst und behandelt. Sind die Symptome verschwunden, kann der Mensch wieder funktionieren. Als Psychoanalytiker stehen Dir bei dieser Vorstellung womöglich die Haare zu Berge. Wo bleibt die Erfassung einer ausführlichen Anamnese, das Bewusstsein für das Symptom als Hinweis auf eine Bewältigungsstrategie einer unbewussten Konfliktkonstellation und der Raum für die Etablierung einer therapeutischen Beziehung, für Ambivalenz und das (Aus-) Halten von Unsicherheit?

Es läuft aber nicht alles reibungslos in der Klinik, und das ist auch gut so. Dein Patient wird mit grosser Wahrscheinlichkeit auf jemanden mit wenig therapeutischer Erfahrung und allenfalls eingeschränkten Sprachkenntnissen treffen. Was für die Institution hinsichtlich der Umsetzung der Regeln ein Problem darstellt, könnte man aber bezüglich Deiner Befürchtung der Klinik als konkrete Maschine auch als Chance verstehen. Hinzu kommt, dass Dein neu eingetretener Patient abgesehen von einem Arzt oder Psychologen auch mit Pflegefachpersonen zu tun haben wird, die unter anderem eine Triangulierung und damit die Voraussetzung für eine Symbolisierung (Grieser, 2010, S. 141) schaffen können.

Die psychopathologische Befunderhebung und die daraus folgende psychiatrische Diagnostik als Grundlage einer Behandlung als Diktat zu bezeichnen, dem Mitarbeiter und Patienten unterworfen sind, greift deutlich zu kurz. Abgesehen davon, dass die Anamnese per Definition eine Voraussetzung für die Diagnostik ist, halte ich diese Regeln und das Erlernen derjenigen für eine wichtige Bedingung, um gerade nicht einem Diktat unterworfen zu sein. Die Institution stellt zwar Regeln auf, ist aber nicht allmächtig. Sie ist selbst Teil eines Systems und muss sich an geltendes Recht halten und psychiatrische Richtlinien berücksichtigen. Nur so erhält sie Legitimation und Mittel, sprich Energie, um die Maschine am Laufen zu halten. Die Regeln (z. B. im Sinne von Diagnosestellung) in der Klinik sind also kein Selbstzweck, sondern vielmehr ein aktueller Konsens auf eine Sprache, der es ermöglicht, u.a. mit Krankenkassen und dem Rechtssystem zu kommunizie- 
ren. Sie sind ein Versuch Realität abzubilden. Im Gegensatz zum Psychotiker, der nach oder bei drohendem Verlust der inneren Struktur durch eine konkretistische Sprache (bspw. durch Neologismen) die Realität fortlaufend selbst erschafft und verändert, versucht die Institution, durch die Sprache eine Grenze zwischen Innen und Aussen zu etablieren, um damit die Voraussetzungen für Symbolisierung in einem dualen Raum (Rom, 2007, S 125 ff.) zu schaffen. Als psychoanalytischer Kliniker kenne ich die Angst, dass das Lernen und Kennen der Regeln mein Denken einengt oder korrumpiert und ich womöglich den Versuch, die Realität abzubilden mit dem konkretistischen Glauben, es handle sich um die Realität, verwechseln könnte. Mit der Bereitschaft, dieses Unbehagen auszuhalten, eröffnet mir die Kenntnis der Regeln Möglichkeiten, mir im Rahmen der Regeln einen Spielraum zu erarbeiten, um nicht in einer konkreten Maschine nur noch zu funktionieren. Wenn ich die Regel und den Grund für deren Errichtung kenne, bin ich in der Lage zu differenzieren und abzuwägen, ob und warum ich mich an die Regel halten will oder eben gerade nicht. Hinzu kommt, dass ich mich als Teil der Institution abhängig von meiner Funktion zur Regelverletzung verhalten muss und kann. Ich kann die Regelverletzung sanktionieren oder aber nach der Bedeutung für den Patienten, den Mitarbeitenden oder die Institution fragen und auf diese Weise durch die Regelverletzung Bedeutung produzieren. Falls mir das Dein Patient als Lüge (Lowtzow, 2008) auslegen würde, nähme ich das gerne in Kauf, solange ich damit einen Spielraum behalte. Die Institution macht die Regeln und der Mensch setzt sie um. Dieser Umstand ist angesichts der Vorstellung des Menschen als mangelhaftes Wesen je nachdem Anlass zur Be(un)ruhigung.

Ich werde das Gefühl nicht los, mir mit diesen allzu allgemeinen Überlegungen etwas zu viel Spielraum zu schaffen. Im konkreten wäre noch viel Differenzierungsbedarf. Zum Beispiel bezüglich der Vorschriften, Auflagen, Abläufe, Gesetze, eigenen Bedürfnisse und inter- und intrapersonellen Dynamiken, die ich bis dahin unter dem Begriff der «Regel» verallgemeinert habe. Und Spielraum bedeutet auch, gegebenenfalls zuviel Möglichkeit und Macht, aber auch Verantwortung zu haben, um je nach Konstellation über die Freiheit und die körperliche Integrität eines Menschen zu entscheiden.

Du als in der Praxis tätiger Arzt und Psychoanalytiker kannst Dich aber auch nicht in die Rolle eines Zuschauers retten. Als Behandler, der Menschen an die Klinikmaschine ankoppelt und nach der Entkoppelung wieder entgegennimmt, bist Du doch auch Teil der Klinik als Symbolisierungsmaschine. Verbunden mit dem Band, das Dinge verbindet, die unverbunden scheinen, wäre doch auch die Praxis als solcher Teil zu denken, der als kleine Institution ähnlichen Gesetzmässigkeiten 
unterworfen ist. Aufgrund der geringeren Grösse ist Deine «Maschine» allenfalls flexibler und selbstbestimmter, aber auch verletzlicher und fehleranfälliger. Verletzlicher, weil Dein Lohn beispielsweise nicht gesichert ist. Deine Existenz hängt also davon ab, ob ein Patient kommt und zahlt. Die Behandlung für den Patienten ist ebenfalls unsicher, da sie ausschliesslich von Deiner Präsenz abhängig ist. Deine Möglichkeit, eine Behandlung zu gewährleisten, ist darüber hinaus durch Deine Aufnahmekapazität und die fehlende Sicherheit im Umgang mit Selbst- oder Fremdgefährdung limitiert. Zudem ist Deine Maschine fehleranfälliger, weil weniger Kontrolle über die Einhaltung von Regeln herrscht. Wäre die Klinik vor diesem Hintergrund möglicherweise auch als subsidiäre Bedingung der Praxis zu denken?

MN: Es ist nur konsequent zu formulieren, dass ich Teil der Maschine bin, die ich mit Sorge betrachte. Und wenn Du über Regeln und den spielerischen Umgang damit schreibst, erinnere ich mich an meinen Anfang in der Praxis, als ich lernen musste die Regeln aufzustellen, die es mir ermöglichen zu arbeiten. Dieser Prozess und seine Kritik haben nicht aufgehört, sondern fordern mich jeden Tag aufs Neue heraus. Ich glaube allerdings nicht, mein Praxisableger der Klinikmaschine sei fehleranfälliger, vielmehr werden die gleichen Probleme wohl unterschiedlich beantwortet. Geht es z. B. um Gewalt, so ist die Position des Klinikers m. E. gefährdeter, diese lustvoll auszuüben. Darin mag eine PsychiatrieTradition fortleben, die Erdheim (2000, S. 168 ff.)schon bei Kraepelin beschreibt: «Der Psychiater ist der <Mächtige> und seine Wissenschaft besteht aus Mitteln, den Patienten $<$ zur Vernunft $>$ zu bringen und sich $<$ die unbedingte Herrschaft über das Gemüt des Kranken zu sichern> (1915, S. 1704) [Hervorhebung im Original]» (Erdheim, 2000, S. 178).

In diesem Kraepelinschen Geist mag es der Klinik passieren, dass sie in den Funktionsmodus einer konkreten Maschine verfällt und die psychischen Realitäten der Menschen in und an der Maschine zermalmt. Die Frage ist nun, ob es der Klinik systemimmanent eingeschrieben ist so zu agieren, oder ob es ihr im Sinne einer «institutionalisierte[n] Abwehr» (Mentzos, 2001, S. 79 ff.) durch die Menschen aufgepfropft wird. Oder trifft auf die Klinik das zu, was Erdheim für die herrschenden Klassen von Gesellschaften mit dem ethnopsychoanalytischen Prozess demonstriert (2000, S. 388 ff.)? Demzufolge gibt es immer das Problem des Umgangs mit Gewalt und damit verbundenen Grössen- und Allmachtsphantasien in der Kultur. Wenn es auf der einen Seite zu einer wünschenswerten Differenzierung einer Gesellschaft kommt, mit klaren Zuständigkeiten und Befugnissen, so ist damit die Gewalt und der damit verbundene Narzissmus nicht gebannt. Vielmehr werden die Herrschenden eines so differenzierten Machtapparates in ihrem Narzissmus 
und der lustvollen, wenngleich nicht offenen, Ausübung von Gewalt gegen die Schwächeren bestärkt. Damit werden sie gleichzeitig aber auch zu Zerstörern der Kultur, die sie erst in diese machtvolle Position versetzt hat. Ich frage mich einfach, wie sich diese unbewussten Mechanismen in der Klinik auswirken, nicht zuletzt da man es dort ja vielfach mit Menschen, z. B. als Patienten, zu tun bekommt, die Selbstzerstörung durch Suizidalität oder mangelnde Selbstfürsorge praktizieren. Wie gross ist da die Versuchung, als Teil der Herrschenden Lust zu gewinnen, indem man auf diese Gewalt auf Seiten der Stärkeren einsteigt? Ich meine diese Verführung erlebt zu haben, als ich mich plötzlich in einer Situation wiederfand, bei der ich mich freiwillig an einer Zwangsmassnahme gegen einen Mann beteiligt habe, bei dem ich davon ausging, er werde trotz seiner Gegenwehr mit einer Spritze mediziert, damit er ausgeschafft werden könne. Bei meinem (im Zuge der Arbeit an diesem Artikel) Jahre späteren Nachfragen konnte mir niemand bestätigen, ob es wirklich so war und es wurde als ungeheuerlich angesehen, würde so etwas in der Klinik getan. Für mich spielt das jedoch keine Rolle, da ich ja in meiner psychischen Realität davon ausging und trotzdem mitmachte, auch wenn dies gegen mein Ethos und Selbstverständnis verstiess. In der Situation selbst fühlte ich mich vor allem verbunden mit meinen Kollegen, als wir den Mann am Boden festhielten.

Welche Mechanismen auch am Werk gewesen sein mögen, für mich bleibt der Umgang mit Gewalt in der Klinikmaschine ein wichtiges Thema. Man könnte meinen, durch meine vergleichsweise schwächere Position in der Einzelpraxis sei ich mit dem Thema Gewalt nicht befasst und könnte dies an die Klinik delegieren. Doch das hiesse sich in eine kitschige Phantasie zu flüchten, die auch schädlich für meine Therapien wäre. Denn auch mir ist durch meinen Status als staatlich anerkannter Therapeut eine Rolle zuerkannt, die mir und meinen Interventionen Macht verleiht und damit auch Gewalt über das Leben meiner Patient*innen. Nehme ich meine Verantwortung ernst, so muss ich akzeptieren, dass auch jede meiner Deutungen etwas Gewalt enthält. Es kommt also nicht darauf an, Gewalt abzulehnen, vielmehr muss ich sie als notwendige Gegebenheit hinnehmen und den Umgang mit ihr lernen. Ich bin eher gezwungen einen produktiven Umgang, wie diesen Artikel, zu finden, der aber nur mit Deiner Hilfe, also im Dialog, entstehen kann.

Das Bier geht zur Neige und «Kliniker» wie «Zuweiser» kehren zurück in ihre Welten, die sie für die Dauer einer Zigarette als immer schon verbunden sehen konnten. Simon Mettler wird sich weiter über aufdringliche oder unerreichbare ambulante Kollegen ärgern, die gerne einmal das Unmögliche von der Klinik erwarten. Michael Niebler wird weiter zweifeln, ob er «seine» Patient*innen 
einer anonymen, kalten und regelindoktrinierten Maschine anvertrauen soll. Die Versuchung wäre gross, ein versöhnliches Ende zu beschwören, doch das hiesse auch auf die Spannung zu verzichten, die ein Verstehen erst ermöglicht. Immerhin ist das nächste Treffen schon verabredet.

\section{Literatur}

Benjamin, W. (2011). Gesammelte Werke (Zweitausendeins Klassiker-Bibliothek). Frankfurt a. M.: Zweitausendeins.

Bob Dylan (Komponist). (1970). The man in me. New Morning(Album): Columbia Records.

Bundesversammlung der Schweizerischen Eidgenossenschaft. (2019). Schweizerisches Zivilgesetzbuch. ZGB. Verfügbar unter https://www.admin.ch/ opc/de/classified-compilation/19070042/index.html\#a426 [16.09.2019].

Deleuze, G. \& Guattari, F. (2014). Anti-Ödipus (Suhrkamp-Taschenbuch Wissenschaft, Bd. 224, 14. Auflage). Frankfurt a. M.: Suhrkamp.

Erdheim, M. (2000). Die gesellschaftliche Produktion von Unbewußtheit. Eine Einführung in den ethnopsychoanalytischen Prozeß (Suhrkamp-Taschenbuch Wissenschaft, Bd. 465, 1. Aufl., [Nachdr.]. Frankfurt a. M.: Suhrkamp.

Foucault, M. (2011). Die Geburt der Klinik. Eine Archäologie des ärztlichen Blicks (Fischer/Taschenbücher] Wissenschaft, Bd. 7400, Ungekürzte Ausg). Frankfurt a. M.: Fischer-Taschenbuch-Verl.

Gaebel, W. \& Zielasek, J. (2011). Äthiopathogenetische Konzepte und Krankheitsmodelle. In H.-J. Möller, G. Laux \& H.-P. Kapfhammer (Hrsg.), Psychiatrie, Psychosomatik, Psychotherapie. Band 1 Allgemeine Psychiatrie (4., S. 81-105). Springer.

Grieser, J. (2010). Der Körper als Dritter-Psychosomatische Triangulierungsprozesse am Beispiel der Adoleszenz. 0032-7034. Verfügbar unter http://psydok. psycharchives.de/jspui/bitstream/20.500.11780/3190/1/59.20102_4_49716. pdf.

Kantonsärztlicher Dienst (Kanton Zürich Gesundheitsdirektion, Hrsg.) (2012). Leitfaden zum neuen Kindes- und Erwachsenenschutzrecht für Ärztinnen und Ärzte, Kanton Zürich Gesundheitsdirektion. Verfügbar unter https://gd.zh. ch/dam/gesundheitsdirektion/direktion/themen/gesundheitsberufe/ aerztin_arzt/leitfadenkindesunderwachsenenschutzrecht/leitfaden_ kindes_und_erwachsenenschutzrecht.pdf.spooler.download.1352 905160537.pdf/leitfaden_kindes_und_erwachsenenschutzrecht.pdf [22.08.2019]. 
Kirchhof, C. (2015). Irritierende Erkenntnis. Zum Stellenwert der Irritation in der Psychoanalyse. Supervision, 33, 17-23.

Lowtzow, D. (Komponist) (2008). Pure Vernunft darfniemals siegen:Buback/Indigo.

Mentzos, S. (2001). Interpersonale und institutionalisierte Abwehr (SuhrkampTaschenbuch Wissenschaft, Bd. 709, Erw. Neuausg., 1. Aufl., [Nachdr.]. Frankfurt a. M.: Suhrkamp.

Mentzos, S. (2004). Warum ist der Wahnkranke sehr oft fast «wahnsüchtig». In P. Hartwich (Hrsg.), Wahn.Definition-Psychodynamik-Therapie (S. 73-74). Sternenfels: Verl. Wiss. und Praxis.

Mettiwetti (Komponist) (2013). Zwei Bier und Zwei Ohren. Nume ine i di gueti Stube (Album).

Niebler, M. (2015). Klinikmaschine. Abschlussarbeit zum Lehrgang Philosophie für Fachleute aus Medizin und Psychotherapie der Universität Zürich, Zürich.

Parin, P. \& Parin-Matthèy, G. (1978). DerWiderspruch im Subjekt. Die Anpassungsmechanismen des Ichs und die Psychoanalyse gesellschaftlicher Prozesse. In S. Drews, R. Klüwer, A. Köhler-Weisker, M. Krüger-Zeul, K. Menne \& H. Vogel (Hrsg.), Provokation und Toleranz. Festschrift für Alexander Mitscherlich zum siebzigsten Geburtstag (1. Auflage, S. 410-435). Frankfurt a. M.: Suhrkamp. Raunig, G. (2008). Tausend Maschinen. Eine kleine Philosophie der Maschine als sozialer Bewegung (Es kommt darauf an, Bd. 7). Wien: Turia + Kant.

Rom, J. (2007). Identitätsgrenzen des Ich. Einblicke in innere Welten schizophrenieund borderlinekranker Menschen. Göttingen: Vandenhoeck \& Ruprecht.

\section{Anmerkungen}

1 Besonderer Dank für die engagierte und kritische Diskussion des Textes gebührt den Teilnehmern des Entresol-Werkstattgesprächs vom 12.12.2019 (Beate Grünebaum, Josef Zwi Guggenheim, Ruth Guggenheim-Tugendhat, Marie-Luise Imholz, Vera Luif, Laura Neri, Reto Pulver, Sophie Schneider, Sonja Stöcklin und Catrina Trippel) sowie Yvonne Schoch, Josi Rom, Heini Schwob und Dani Strassberg.

\section{Angaben zu den Autoren}

Michael Niebler, Dr. med., 1983, Facharzt für Psychiatrie und Psychotherapie FMH in freier Praxis, zweifelte an derWahl seines Faches bis er herausfand, dass es unter den Psychiatern auch Philosophen gibt. www.michaelniebler.ch

Simon Mettler, lic. phil., 1982, Eidg. anerkannter Psychoanalytischer Psychotherapeut, Mitglied des PSZ, Leitender Psychologe Erwachsenen-Akutbereich ipw Hard, wechselte einst von der Soteria in die Akutpsychiatrie und versteht erst allmählich, warum er diese Entscheidung bis heute nicht bereut. 